\title{
Analysis of Flapping Motion of Rotors of Small Coaxial Helicopter
}

\author{
By Shigeru Sunada, ${ }^{1,2)}$ Hiroki Sumino, ${ }^{1)}$ Atsushi Matsue ${ }^{1) * *}$ and Hiroshi ToKutaKe ${ }^{1)}$ \\ ${ }^{1)}$ Department of Aerospace Engineering, Osaka Prefecture University, Osaka, Japan \\ ${ }^{2)}$ PRESTO, Japan Science and Technology Corporation, Kawaguchi, Japan
}

(Received August 31st, 2005)

\begin{abstract}
We analyzed a small coaxial helicopter developed for entertainment in 2002. The upper and lower rotors are rigid rotors. The upper rotor is connected to a stabilizer bar. The cyclic pitch of the upper rotor is controlled by the stabilizer bar when the attitude of the helicopter is varied. The angle between the upper rotor and the stabilizer bar is $41^{\circ}$. The cyclic pitch of the lower rotor is controlled by servo motors and the inputs of the cyclic pitch from the servo motors are at the azimuth angles of $45^{\circ}, 225^{\circ}$ and $135^{\circ}, 315^{\circ}$. This paper clarifies how the angle between the upper rotor and its stabilizer bar and the azimuth angles of the inputs of the cyclic pitch to the lower rotor are determined.
\end{abstract}

Key Words: Flapping Motion, Cyclic Pitch Control, Rigid Rotor, Stabilizer Bar

\section{Nomenclature}

$A, \bar{B}, C:$ matrices indicated in Eq. (12)

a: lift slope

$B$ : tip loss factor

$C_{\mathrm{T}}, C_{\mathrm{Q}}:$ thrust and torque coefficients

$C_{1}, C_{\mathrm{d}}$ : lift and drag coefficients of blade element

$C_{\mathrm{d} 0}$ : averaged value of $C_{\mathrm{d}}$

$c$ : chord length

$\bar{c}$ : averaged chord length

$D$ : phase difference between $\beta$ and $\theta$

$d$ : distance between upper and lower rotors

$F$ : force applied on blade

$G$ : ratio between $\Delta \beta$ and $\Delta \theta$

$g$ : gravity acceleration

$h$ : hub location from center of gravity

$I_{\beta}$ : moment of inertia of blade about virtual flapping hinge, $=\int_{r_{\beta}}^{R}\left(r-r_{\beta}\right)^{2} m \mathrm{~d} r$

$i$ : inclination of rotor shaft

$K_{0}, K_{1}, K_{1}{ }^{\prime}, K_{1}{ }^{\prime \prime}$,

$K_{2}, K_{2}{ }^{\prime}, K_{3}$ : constants indicated in Table 2

$k_{\beta}, k_{\dot{\beta}}$ : spring stiffness and damping coefficient

$M_{1} \sim M_{6}, \bar{M}_{1}$, for flapping angles, indicated by Eq. (5)

$\bar{M}_{2}, \bar{M}_{4}, \bar{M}_{6}$ : coefficients in flapping equation, Eq. (7) or Eq. (11)

$M_{\mathrm{A}}$ : aerodynamic flapwise moment given by Eqs. (3) and (8)

$M_{\mathrm{I}}$ : inertial flapwise moment given by Eqs. (4) and (9)

$M_{\mathrm{m}}$ : mechanical flapwise moment given by Eqs. (5) and (10)

$m$ : mass of blade element for unit width

$P$ : angular velocity of helicopter fuselage around $X$ axis
$Q$ : angular velocity of helicopter fuselage around $Y$ axis

$R$ : blade radius

$r$ : radius of blade element

$r_{\beta}$ : radius of virtual flapping hinge location

$U_{\mathrm{P}}$ : perpendicular component of inflow velocity to blade element

$U_{\mathrm{T}}$ : tangential component of inflow velocity to blade element

$V$ : forward velocity

$v$ : induced velocity $=v_{0}+v_{\mathrm{c}} \cos \varphi+v_{\mathrm{s}} \sin \varphi$ $W_{\beta} \bar{r}=\int_{r_{\beta}}^{R} m g\left(r-r_{\beta}\right) \mathrm{d} r$

$x, x_{\beta}: r / R, r_{\beta} / R$

$\alpha$ : angle of attack

$\alpha_{0}$ : angle of attack for zero lift

$\beta$ : blade flapping angle measured from plane normal to shaft

$\beta_{0}$ : coning angle

$\bar{\beta}_{0}$ : preconing angle

$\varphi$ : azimuth angle

$\gamma:$ lock number

$\lambda_{0}, \lambda_{\mathrm{c}}, \lambda_{\mathrm{s}}=\left(V \sin i+v_{0}\right) / R \Omega, v_{\mathrm{c}} / R \Omega, v_{\mathrm{s}} / R \Omega$

$\mu=V \cos i / R \Omega$

$\theta$ : blade pitch angle

$\bar{\theta}:$ approximation of $\theta$ when $\theta_{\mathrm{c}}=\theta_{\mathrm{s}}=0$

$\theta_{\mathrm{t}}$ : blade twist angle

$\rho$ : air density

$\sigma$ : solidity

$\Omega$ : rotational speed

$\delta$ : bending deformation of blade

$\Delta \beta=\beta-\beta_{0}$

$\Delta \bar{\beta}=\sqrt{\beta_{\mathrm{c}}{ }^{2}+\beta_{\mathrm{s}}{ }^{2}}$

$\Delta \theta=\theta-\theta_{0}(r=0)$

$\Delta \bar{\theta}=\sqrt{\theta_{\mathrm{c}}^{2}+\theta_{\mathrm{s}}^{2}}$

$\zeta$ : angle between stabilizer bar and upper rotor 


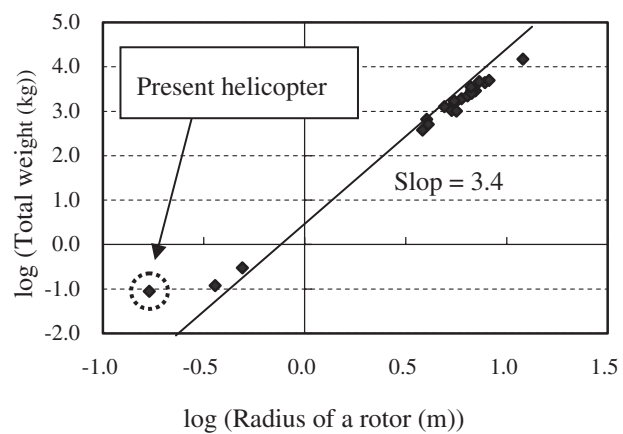

Fig. 1. Relation between radius and weight of various helicopters.

\section{Subscripts}

1, s, u: lower rotor, stabilizer bar, upper rotor

\section{Introduction}

A small coaxial helicopter ${ }^{1}$ was released for entertainment in 2002 with the following features. (i) The upper and lower rotors are rigid. The shape of the upper rotor is same as the lower rotor. (ii) The upper rotor is connected to a stabilizer bar at an angle of $41^{\circ}{ }^{2)}$ The cyclic pitch angle of the upper rotor is varied only by this stabilizer bar. (iii) The cyclic pitch control for the lower rotor is performed by servo motors and the inputs are at the azimuth angles of $45^{\circ}, 225^{\circ}$ and $135^{\circ}, 315^{\circ}$. (iv) The upper and lower rotors do not have collective pitch control. Instead of collective pitch control, the rotational speeds of both rotors are controlled to vary thrust. (v) Yaw is controlled by the different revolution speed of each rotor, referring to angular speed measured by a rate gyro. (vi) The relationship between the radius and weight of various helicopter designs is shown in Fig. 1, which shows the data for all helicopters except the helicopter analyzed in this paper are on the same line with a slope of 3.4. The present helicopter is not on this line and is very heavy for its radius. This means that the rotational speed of the rotors of the present helicopter is large for its size compared to the other helicopters.

This paper analyzes the angle between the stabilizer bar and the upper rotor stated in (ii), and the azimuth angles of the inputs of the cyclic pitch to the lower rotor stated in (iii). Hovering flight is considered because the coaxial helicopter flies mainly at a low speed.

\section{Rotor Characteristics}

\section{Measurement of aerodynamic characteristics}

The physical parameters of the helicopter are shown in Table 1. The distributions of pitch angle (when $\theta_{\mathrm{c}}=\theta_{\mathrm{s}}=$ 0 ) and chord length for the upper and lower rotors are shown
Table 1. Physical data of the helicopter.

\begin{tabular}{lc}
\multicolumn{1}{c}{ Items } & Value \\
\hline Body weight, $W[\mathrm{~kg}]$ & 0.16 \\
Rotor radius, $R[\mathrm{~m}]$ & 0.172 \\
Averaged chord length, $c[\mathrm{~m}]$ & 0.022 \\
Number of blades, $b$ & 2 \\
Lock number, $\gamma$ & $3.4 a(a:$ lift slope $)$ \\
Rotor angular velocity, $\Omega$ [rad $/ \mathrm{s}]$ & 157 \\
Inertia of moment about virtual & $6.9 \times 10^{-6}$ \\
$\quad$ flapping hinge, $I_{\beta}\left[\mathrm{kgm}{ }^{2}\right]$ & 0.027 \\
Radius of virtual flapping hinge & -5 \\
$\quad$ location, $r_{\beta}[\mathrm{m}]$ & -1.5 \\
Blade twist, $\theta_{\mathrm{t}}[\mathrm{deg}]$ & $7.9 \times 10^{-4}$ \\
Preconing angle, $\bar{\beta}_{0}[\mathrm{deg}]$ & \\
$W_{\beta} \bar{r}\left[\mathrm{kgm}{ }^{2} / \mathrm{s}^{2}\right]$ & 0.4 \\
Non-dimensional distance $^{\text {between upper and lower rotors, } d / R}$ & \\
\end{tabular}

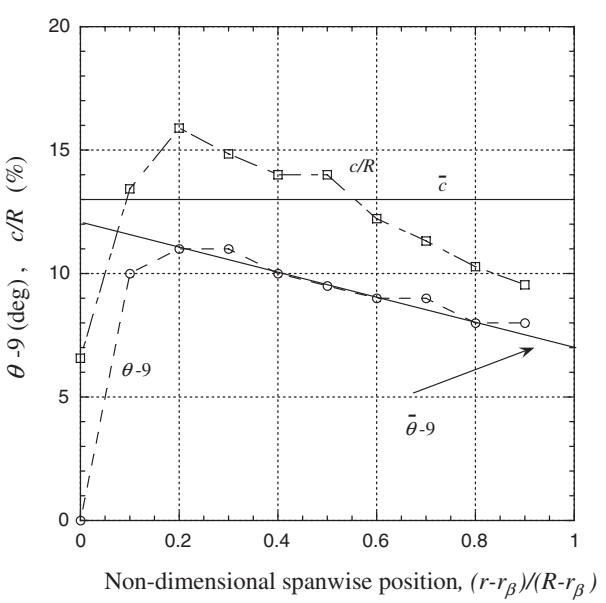

Fig. 2. Distributions of geometrical angle of attack $\theta$ and chord length $c / R . \bar{\theta}$ is the approximation for $\theta$, and $\bar{c}$ is the averaged chord length.

in Fig. 2. The averaged chord length $\bar{c}$ is $0.13 R=22 \mathrm{~mm}$. The pitch angle $\theta$ is approximated by $\bar{\theta}=\left\{21-5\left(r-r_{\beta}\right) /\right.$ $\left.\left(R-r_{\beta}\right)\right\}(\mathrm{deg})$. A big difference is observed between $\theta$ and $\bar{\theta}$ near the blade root. However, this difference does not cause a big error in estimating thrust and torque because the aerodynamic force acting near the blade hinge is small.

Figures 3(a) and (b) show the measured relationship between $\theta\left(r=r_{\beta}\right)$ and $C_{\mathrm{T}} / \sigma$ for a single rotor $^{3)}$ and that between $\theta\left(r=r_{\beta}\right)$ and $C_{\mathrm{Q}} / \sigma$ by the open circles, respectively. In these figures, the calculated values are also shown by filled diamonds. These calculations were made using the annular momentum and blade element theories. ${ }^{4)}$ In the calculations, the approximated collective pitch at the blade hinge $\bar{\theta}$ was used and the following airfoil characteristics are assumed.

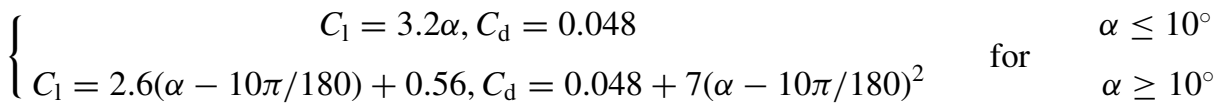




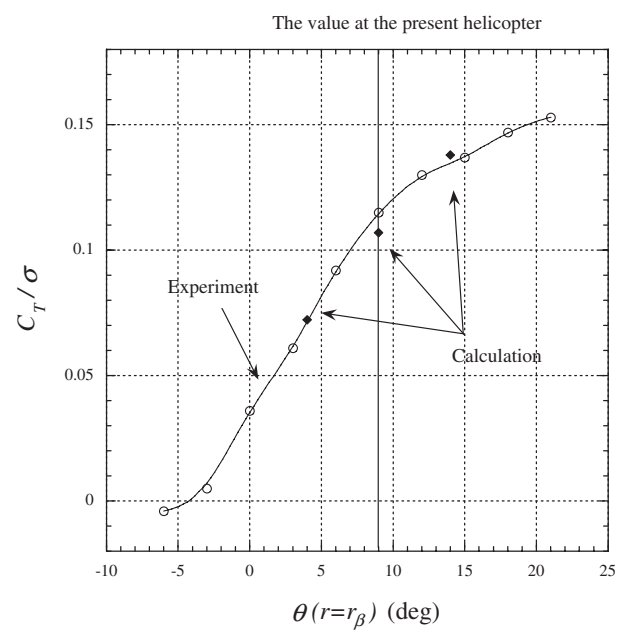

(a)

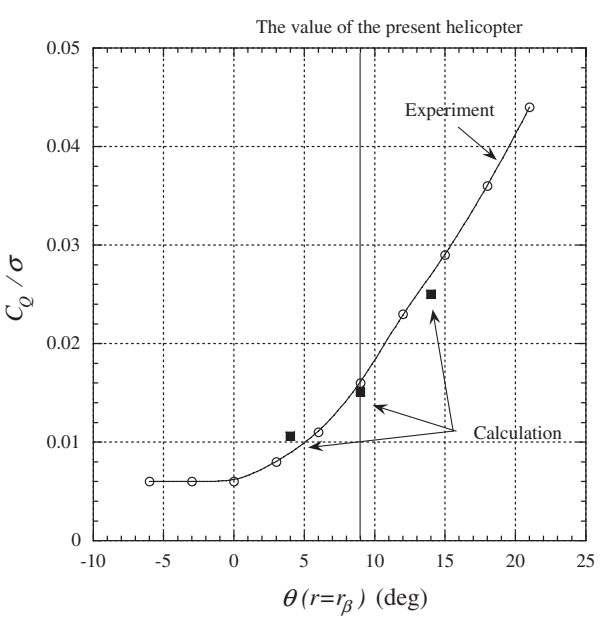

(b)

Fig. 3. Aerodynamic characteristics of single rotor.

(a) Thrust coefficient $C_{\mathrm{T}} / \sigma$, (b) Torque coefficient $C_{\mathrm{Q}} / \sigma$.

Good agreements between the measured and calculated values are seen in Fig. 3, meaning the airfoil characteristics assumed by Eqs. (1a) and (1b) are relevant.

\section{Measurement of elastics characteristics}

The rigid blades of the helicopter are treated as equivalent hinged rigid blades ${ }^{5)}$ in the later analysis. Flapping motion was observed around the blade root, so the virtual flapping hinge is coincident with the blade root. The spring restraint about the virtual flapping hinge was estimated as follows: The deformation $\delta$ was measured when the load $F$ was applied to the lower side of $r-r_{\beta}=0.138 \mathrm{~m}$ of the rotor. The value of $k_{\beta}$ was estimated by beam theory as $k_{\beta}=$ $F\left(r-r_{\beta}\right)^{2} / \delta$. The estimated value of $k_{\beta}$ is $0.07 \mathrm{Nm}$.

\section{Flapping motion}

The cyclic pitch to the lower rotor is made at $\varphi=45^{\circ}$, $225^{\circ}$ by one servo motor. For hovering flight, this cyclic pitch generates lateral flapping motion with largest absolute value at $\varphi \approx 90^{\circ}, 270^{\circ}$. The cyclic pitch with largest absolute value at $\varphi=135^{\circ}, 315^{\circ}$ by the other servo motor generates longitudinal flapping motion with largest absolute value at $\varphi \approx 180^{\circ}, 360^{\circ}$. Consequently, we can expect that for hovering flight, the phase difference between $\beta$ and $\theta$, $D$, is approximately $45^{\circ}$ for the lower rotor.

For hovering flight, the lateral and longitudinal flapping motion can be controlled separately by two cyclic pitch inputs via the servo motors, making flight control much easier. However, the phase difference between $\beta$ and $\theta$ depends on forward velocity. ${ }^{6-8)}$ So for forward flight, the pilot must input both the lateral and longitudinal cyclic pitch just to make either lateral or longitudinal flapping motion of the lower rotor.

The cyclic pitch of the upper rotor is made with the flapping motion of the stabilizer bar when the attitude of the helicopter is varied. The tip path plane of the upper rotor is inclined left and right for $P>0$ and $P<0$, respectively. It is inclined forwards and backwards for $Q>0$ and $Q<0$, respectively. Therefore, the flapping motion of the upper rotor caused by the cyclic pitch from the stabilizer bar decreases the attitude disturbance of the fuselage. The flapping motions of the upper rotor is related to the angle between it and the stabilizer bar.

\section{Mathematical Model of Flapping Motion}

The equation for blade flapping motion when a fuselage has the pitching and rolling motions, $P$ and $Q$ shown in Fig. 4 is:

$$
M_{\mathrm{A}}+M_{\mathrm{I}}+M_{\mathrm{m}}=0 .
$$

The values of $M_{\mathrm{A}}, M_{\mathrm{I}}$ and $M_{\mathrm{m}}$ are given as follows:

$$
\begin{aligned}
M_{\mathrm{A}} / & \frac{1}{2} \rho(R \Omega)^{2} \\
= & \beta^{2}\left[a \int_{r_{\beta}}^{B R}\left(D_{0}^{2} \theta+A_{0} D_{0}\right)\left(r-r_{\beta}\right) c \mathrm{~d} r\right. \\
& \left.-\int_{r_{\beta}}^{R} A_{0} D_{0} C_{\mathrm{d} 0}\left(r-r_{\beta}\right) c \mathrm{~d} r\right]
\end{aligned}
$$

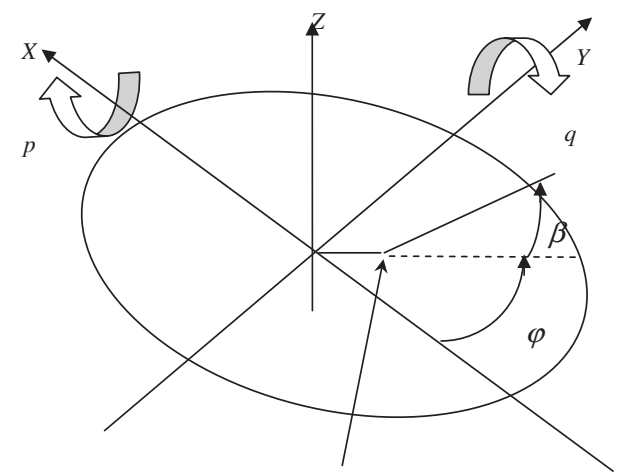

Virtual flapping hinge

Fig. 4. Definitions of wing motions and body fixed coordinate system $X-Y-Z$. 


$$
\begin{aligned}
& +\beta\left[a \int_{r_{\beta}}^{B R}\left(2 D_{0} E_{0} \theta+A_{0} E_{0}+C_{0} D_{0}\right)\left(r-r_{\beta}\right) c \mathrm{~d} r\right. \\
& \left.-\int_{r_{\beta}}^{R}\left(A_{0} E_{0}+C_{0} D_{0}\right) C_{\mathrm{d} 0}\left(r-r_{\beta}\right) c \mathrm{~d} r\right] \\
& +\dot{\beta}\left[a \int_{r_{\beta}}^{B R} B_{0} E_{0}\left(r-r_{\beta}\right) c \mathrm{~d} r\right. \\
& \left.-\int_{r_{\beta}}^{R} B_{0} E_{0} C_{\mathrm{d} 0}\left(r-r_{\beta}\right) c \mathrm{~d} r\right] \\
& +\dot{\beta}_{\beta}\left[a \int_{r_{\beta}}^{B R} B_{0} D_{0}\left(r-r_{\beta}\right) c \mathrm{~d} r\right. \\
& \left.-\int_{r_{\beta}}^{R} B_{0} D_{0} C_{\mathrm{d} 0}\left(r-r_{\beta}\right) c \mathrm{~d} r\right] \\
& +a \int_{r_{\beta}}^{B R}\left(E_{0}^{2} \theta+C_{0} E_{0}\right)\left(r-r_{\beta}\right) c \mathrm{~d} r \\
& -\int_{r_{\beta}}^{R} C_{0} E_{0} C_{\mathrm{d} 0}\left(r-r_{\beta}\right) c \mathrm{~d} r
\end{aligned}
$$

where

$$
\begin{aligned}
& A_{0}=-\frac{h}{R}\left(\frac{P}{\Omega} \sin \varphi+\frac{Q}{\Omega} \cos \varphi\right)-\mu \cos \varphi \cos i, \\
& B_{0}=-\frac{1}{\Omega}\left(x-x_{\beta}\right), \\
& C_{0}=x\left(\frac{P}{\Omega} \sin \varphi+\frac{Q}{\Omega} \cos \varphi\right) \\
& -\mu \sin i-\lambda_{0}-\lambda_{\mathrm{c}} \cos \varphi-\lambda_{\mathrm{s}} \sin \varphi \\
& D_{0}=-\left(x-x_{\beta}\right)\left(\frac{Q}{\Omega} \sin \varphi-\frac{P}{\Omega} \cos \varphi\right), \\
& E_{0}=\mu \cos i \sin \varphi+x+\frac{h}{R}\left(\frac{Q}{\Omega} \sin \varphi-\frac{P}{\Omega} \cos \varphi\right) . \\
& M_{\mathrm{I}}=-\dot{\beta} \beta \int_{r_{\beta}}^{R} \mathrm{~d} m\left(r-r_{\beta}\right)\left\{-r_{\beta}(P \sin \varphi+Q \cos \varphi)+V \sin i\right\} \\
& -\beta \int_{r_{\beta}}^{R} \mathrm{~d} m\left(r-r_{\beta}\right)\{h(\dot{P} \sin \varphi+\dot{Q} \cos \varphi) \\
& -(P \sin \varphi+Q \cos \varphi) V \sin i+r \Omega^{2} \\
& \left.-(-P \cos \varphi+Q \sin \varphi)^{2} r+\left(P^{2}+Q^{2}\right) r_{\beta}\right\} \\
& -\ddot{\beta} \int_{r_{\beta}}^{R} \mathrm{~d} m\left(r-r_{\beta}\right)^{2} \\
& +\beta^{2} \int_{r_{\beta}}^{R} \mathrm{~d} m \Omega\left(r-r_{\beta}\right)^{2}(-P \cos \varphi+Q \sin \varphi) \\
& -\int_{r_{\beta}}^{R} \mathrm{~d} m\left(r-r_{\beta}\right)\left\{h\left(P^{2}+Q^{2}\right)+V Q \cos i\right. \\
& -r(\dot{P} \sin \varphi+2 P \Omega \cos \varphi+\dot{Q} \cos \varphi-2 Q \Omega \sin \varphi)\}
\end{aligned}
$$

$$
M_{\mathrm{m}}=-k_{\beta}\left(\beta-\overline{\beta_{0}}\right)-k_{\dot{\beta}} \dot{\beta} .
$$

The blade element theory is used to obtain $M_{\mathrm{A}}$. The perpendicular and tangential components of inflow velocity to the blade element, $U_{\mathrm{P}}$ and $U_{\mathrm{T}}$, are given by

$$
\left\{\begin{aligned}
\frac{U_{\mathrm{P}}}{R \Omega}= & \left(-\beta \frac{h}{R \Omega}+\frac{x}{\Omega}\right)(P \sin \varphi+Q \cos \varphi) \\
& -\mu \beta \cos \varphi \cos i-\mu \sin i-\frac{\dot{\beta}}{\Omega}\left(x-x_{\beta}\right) \\
& -\lambda_{0}-\lambda_{\mathrm{c}} \cos \varphi-\lambda_{\mathrm{s}} \sin \varphi \\
\frac{U_{\mathrm{T}}}{R \Omega}= & \left(\frac{h}{R \Omega}-\frac{x-x_{\beta}}{\Omega} \beta\right)(Q \sin \varphi-P \cos \varphi) \\
& +\mu \cos i \sin \varphi+x
\end{aligned}\right.
$$

And the aerodynamic characteristics of blade element are given by $C_{1}=a \alpha$ and $C_{\mathrm{d}}=C_{\mathrm{d} 0}$. For $C_{1}=a\left(\alpha-\alpha_{0}\right)$ such as Eq. (1b), analysis is possible when $\theta_{0}$ is replaced by $\theta_{0}-\alpha_{0}$.

Substituting Eqs. (3)-(5) into Eq. (2) gives the following equation.

$$
M_{1} \ddot{\beta}+M_{2} \dot{\beta}+M_{3} \dot{\beta} \beta+M_{4} \beta+M_{5} \beta^{2}+M_{6}=0
$$

This equation includes the non-linear terms and should be solved numerically. To linearize this equation, the following assumptions (A)-(G) are adopted for Eqs. (3)-(6).

(A) The flapping angle $\beta$ and pitching angle $\theta$ are assumed to be given by $\beta=\beta_{0}(t)+\beta_{\mathrm{c}}(t) \cos \varphi+\beta_{\mathrm{s}}(t) \sin \varphi$ and $\theta=\theta_{0}+\theta_{\mathrm{c}} \cos \varphi+\theta_{\mathrm{s}} \sin \varphi+\theta_{\mathrm{t}} \frac{r}{R}$, respectively.

(B) $\beta \ll h / r_{\beta}, \beta \gg r / h, \beta^{2} \ll 1, \beta \dot{\beta} \ll 1, P / \Omega, Q / \Omega \ll 1$

(C) The $U_{\mathrm{P}}$ and $U_{\mathrm{T}}$ are approximated by given by

$$
\left\{\begin{aligned}
U_{\mathrm{p}} / R \Omega= & x \sin \varphi\left(-\frac{\dot{\beta}_{\mathrm{s}}}{\Omega}+\frac{P}{\Omega}+\beta_{\mathrm{c}}\right) \\
& -x \cos \varphi\left(\frac{\dot{\beta}_{\mathrm{c}}}{\Omega}-\frac{Q}{\Omega}+\beta_{\mathrm{s}}\right) \\
& -\mu \beta \cos \varphi-\lambda_{0}-\lambda_{\mathrm{c}} \cos \varphi-\lambda_{\mathrm{S}} \sin \varphi \\
U_{\mathrm{T}} / R \Omega= & \mu \sin \varphi+x
\end{aligned}\right.
$$

And

$$
U_{\mathrm{T}}^{2}+U_{\mathrm{P}}^{2} \approx U_{\mathrm{T}}^{2}
$$

(D) The chord length $c$ at any span-wise position is assumed to be the averaged value of $c, \bar{c}$.

(E) The moment due to aerodynamic force acting on the blade element between 0 and $r_{\beta}$ is ignored.

(F) $c_{\mathrm{d}}=0$

(G) The $M_{\mathrm{A}}, M_{\mathrm{I}}$ and $M_{\mathrm{m}}$ include only a constant and terms proportional to $\cos \varphi$ and $\sin \varphi$.

With these assumptions, $M_{\mathrm{A}}, M_{\mathrm{I}}$ and $M_{\mathrm{m}}$ can be expressed as follows: 


$$
\begin{aligned}
& M_{\mathrm{A}}=\frac{1}{2} \rho a \bar{c} R^{4} \Omega^{2}\left[\left\{\frac{B^{4}}{4}-\frac{B^{3}}{3} x_{\beta}+\mu^{2}\left(\frac{B^{2}}{4}-\frac{B}{2} x_{\beta}\right)\right\} \theta_{0}\right. \\
& +\mu\left(\frac{B^{3}}{3}-\frac{B^{2}}{2} x_{\beta}\right) \theta_{\mathrm{s}}+\left\{\frac{B^{5}}{5}-\frac{B^{4}}{4} x_{\beta}+\mu^{2}\left(\frac{B^{3}}{6}-\frac{B^{2}}{4} x_{\beta}\right)\right\} \theta_{\mathrm{t}} \\
& -\left(\frac{B^{4}}{4}-\frac{2 B^{3}}{3} x_{\beta}+\frac{B^{2}}{2} x_{\beta}^{2}\right) \frac{\dot{\beta}_{0}}{\Omega}-\mu\left(\frac{B^{3}}{6}-\frac{B^{2}}{2} x_{\beta}+\frac{B}{2} x_{\beta}^{2}\right)\left(\frac{\dot{\beta}_{\mathrm{s}}}{\Omega}-\frac{P}{\Omega}\right) \\
& -\mu\left(\frac{B^{2}}{4} x_{\beta}-\frac{B}{2} x_{\beta}^{2}\right) \frac{\beta_{\mathrm{c}}}{\Omega}-\lambda_{0}\left(\frac{B^{3}}{3}-\frac{B^{2}}{2} x_{\beta}\right)-\mu \lambda_{\mathrm{s}}\left(\frac{B^{2}}{4}-\frac{B}{2} x_{\beta}\right) \\
& +\left\{\left(\frac{B^{4}}{4}-\frac{B^{3}}{3} x_{\beta}+\frac{\mu^{2} B^{2}}{8}-\frac{\mu^{2} B}{4} x_{\beta}\right) \theta_{\mathrm{c}}\right. \\
& -\left(\frac{B^{4}}{4}-\frac{2 B^{3}}{3} x_{\beta}+\frac{B^{2}}{2} x_{\beta}^{2}\right)\left(\frac{\dot{\beta}_{\mathrm{c}}}{\Omega}-\frac{Q}{\Omega}\right)-\mu\left(\frac{B^{3}}{3}-\frac{B^{2}}{2} x_{\beta}\right) \beta_{0} \\
& \left.-\left(\frac{B^{4}}{4}-\frac{2 B^{3}}{3} x_{\beta}+\frac{B^{2}}{2} x_{\beta}^{2}+\frac{\mu^{2} B^{2}}{8}-\frac{\mu^{2} B}{4} x_{\beta}\right) \beta_{\mathrm{s}}-\lambda_{\mathrm{c}}\left(\frac{B^{3}}{3}-\frac{B^{2}}{2} x_{\beta}\right)\right\} \cos \varphi \\
& +\left\{\mu\left(\frac{2 B^{3}}{3}-B^{2} x_{\beta}\right) \theta_{0}+\left(\frac{B^{4}}{4}-\frac{B^{3}}{3} x_{\beta}+\frac{3 \mu^{2} B^{2}}{8}-\frac{3 \mu^{2} B}{4} x_{\beta}\right) \theta_{\mathrm{s}}\right. \\
& +\mu\left(\frac{B^{4}}{2}-\frac{2 B^{3}}{3} x_{\beta}\right) \theta_{\mathrm{t}}-\mu\left(\frac{B^{3}}{3}-B^{2} x_{\beta}+B x_{\beta}^{2}\right) \frac{\dot{\beta}_{0}}{\Omega}-\left(\frac{B^{4}}{4}-\frac{2 B^{3}}{3} x_{\beta}+\frac{B^{2}}{2} x_{\beta}^{2}\right)\left(\frac{\dot{\beta}_{\mathrm{s}}}{\Omega}-\frac{P}{\Omega}\right) \\
& \left.\left.+\left(\frac{B^{4}}{4}-\frac{2 B^{3}}{3} x_{\beta}+\frac{B^{2}}{2} x_{\beta}^{2}-\frac{\mu^{2} B^{2}}{8}+\frac{\mu^{2} B}{4} x_{\beta}\right) \beta_{\mathrm{c}}-\mu \lambda_{0}\left(\frac{B^{2}}{2}-B x_{\beta}\right)-\lambda_{\mathrm{s}}\left(\frac{B^{3}}{3}-\frac{B^{2}}{2} x_{\beta}\right)\right\} \sin \varphi\right] \\
& M_{\mathrm{I}}=-I_{\beta}\left\{\ddot{\beta}_{0}+\left(\ddot{\beta}_{\mathrm{c}}+2 \dot{\beta}_{\mathrm{s}} \Omega-\beta_{\mathrm{c}} \Omega^{2}\right) \cos \varphi+\left(\ddot{\beta}_{\mathrm{s}}-2 \dot{\beta}_{\mathrm{c}} \Omega-\beta_{\mathrm{s}} \Omega^{2}\right) \sin \varphi\right\} \\
& +\left(I_{\beta}+r_{\beta} \frac{W_{\beta} \bar{r}}{g}\right)\left\{-\beta_{0} \Omega^{2}+\left(2 P \Omega+\dot{Q}-\beta_{\mathrm{c}} \Omega^{2}\right) \cos \varphi+\left(-2 Q \Omega+\dot{P}-\beta_{\mathrm{s}} \Omega^{2}\right) \sin \varphi\right\} \\
& -\frac{W_{\beta} \bar{r}}{g} V\left\{Q \cos i-\left(\frac{1}{2} P \beta_{\mathrm{s}}+\frac{1}{2} Q \beta_{\mathrm{c}}\right) \sin i-Q \beta_{0} \sin i \cos \varphi-P \beta_{0} \sin i \sin \varphi\right\} \\
& M_{\mathrm{m}}=-k_{\beta}\left(\beta-\bar{\beta}_{0}\right)-k_{\dot{\beta}} \dot{\beta} \\
& =-k_{\beta} \beta_{0}-k_{\dot{\beta}} \dot{\beta}_{0}+k_{\beta} \bar{\beta}_{0}+\left(-k_{\beta} \beta_{\mathrm{c}}-k_{\dot{\beta}} \dot{\beta}_{\mathrm{c}}-k_{\dot{\beta}} \beta_{\mathrm{s}} \Omega\right) \cos \varphi+\left(-k_{\beta} \beta_{\mathrm{s}}-k_{\dot{\beta}} \dot{\beta}_{\mathrm{s}}+k_{\dot{\beta}} \beta_{\mathrm{c}} \Omega\right) \sin \varphi .
\end{aligned}
$$

Substituting Eqs. (8)-(10) into Eq. (7), the following linear equation is obtained.

$$
\bar{M}_{1} \ddot{\beta}+\bar{M}_{2} \dot{\beta}+\bar{M}_{4} \beta+\bar{M}_{6}=0
$$

Equations similar to Eq. (11) are shown in Refs. 9) and 10). However, the flapping equations in Ref. 9) are for when $V=0$ and blade twist is ignored in Ref. 10).

From Eq. (11), the relation between $\beta_{0}, \beta_{\mathrm{c}}, \beta_{\mathrm{s}}$ and $\theta_{0}, \theta_{\mathrm{c}}$, $\theta_{\mathrm{s}}$ in a steady terminal state of flapping motion is given as follows:

$$
A\left(\begin{array}{c}
\beta_{0} \\
\beta_{\mathrm{c}} \\
\beta_{\mathrm{s}}
\end{array}\right)+\bar{B}\left(\begin{array}{c}
\theta_{0} \\
\theta_{\mathrm{c}} \\
\theta_{\mathrm{s}}
\end{array}\right)+C=\mathbf{0}
$$

The components of the matrices $A, \bar{B}$ and $C$ are shown in Table 2. For hovering flight, the flapping angles, $\beta_{0}, \beta_{\mathrm{c}}$, $\beta_{\mathrm{s}}$, can be obtained by solving Eq. (12) as follows:

$$
\beta_{0}=\frac{\theta_{0} K_{1}-\lambda_{0} K_{2}+\theta_{\mathrm{t}}(\gamma / 2)\left(B^{5} / 5-x_{\beta} B^{4} / 4\right)+\left(k_{\beta} / I_{\beta} \Omega^{2}\right) \bar{\beta}_{0}}{1+W_{\beta} \bar{r} r_{\beta} / g I_{\beta}+k_{\beta} / I_{\beta} \Omega^{2}}
$$

$$
\begin{aligned}
\beta_{\mathrm{c}}= & {\left[( W _ { \beta } \overline { r } r _ { \beta } / g I _ { \beta } + k _ { \beta } / I _ { \beta } \Omega ^ { 2 } ) \left\{\theta_{\mathrm{c}} K_{1}-\lambda_{\mathrm{c}} K_{2}\right.\right.} \\
& \left.+K_{1}{ }^{\prime}(Q / \Omega)+\left(1+W_{\beta} \bar{r} r_{\beta} / g I_{\beta}\right)(2 P / \Omega)\right\} \\
& +\left(K_{1}{ }^{\prime}+k_{\dot{\beta}} / I_{\beta} \Omega\right)\left\{-\theta_{\mathrm{s}} K_{1}+\lambda_{\mathrm{s}} K_{2}\right. \\
& \left.\left.-K_{1}{ }^{\prime}(P / \Omega)+\left(1+W_{\beta} \bar{r} r_{\beta} / g I_{\beta}\right)(2 Q / \Omega)\right\}\right] \\
& \cdot\left\{\left(K_{1}{ }^{\prime}+k_{\dot{\beta}} / I_{\beta} \Omega\right)^{2}+\left(W_{\beta} \bar{r} r_{\beta} / g I_{\beta}+k_{\beta} / I_{\beta} \Omega^{2}\right)^{2}\right\}^{-1} \\
\beta_{\mathrm{s}}= & {\left[( K _ { 1 } { } ^ { \prime } + k _ { \dot { \beta } } / I _ { \beta } \Omega ) \left\{\theta_{\mathrm{c}} K_{1}-\lambda_{\mathrm{c}} K_{2}\right.\right.} \\
& \left.+K_{1}{ }^{\prime}(Q / \Omega)+\left(1+W_{\beta} \bar{r} r_{\beta} / g I_{\beta}\right)(2 P / \Omega)\right\} \\
& -\left(W_{\beta} \bar{r} r_{\beta} / g I_{\beta}+k_{\beta} / I_{\beta} \Omega^{2}\right)\left\{-\theta_{\mathrm{s}} K_{1}+\lambda_{\mathrm{s}} K_{2}\right. \\
& \left.\left.-K_{1}{ }^{\prime}(P / \Omega)+\left(1+W_{\beta} \bar{r} r_{\beta} / g I_{\beta}\right)(2 Q / \Omega)\right\}\right] \\
& \cdot\left\{\left(K_{1}{ }^{\prime}+k_{\dot{\beta}} / I_{\beta} \Omega\right)^{2}+\left(W_{\beta} \bar{r} r_{\beta} / g I_{\beta}+k_{\beta} / I_{\beta} \Omega^{2}\right)^{2}\right\}^{-1}
\end{aligned}
$$


Table 2. Components of matrices $A, \bar{B}$ and $C$.

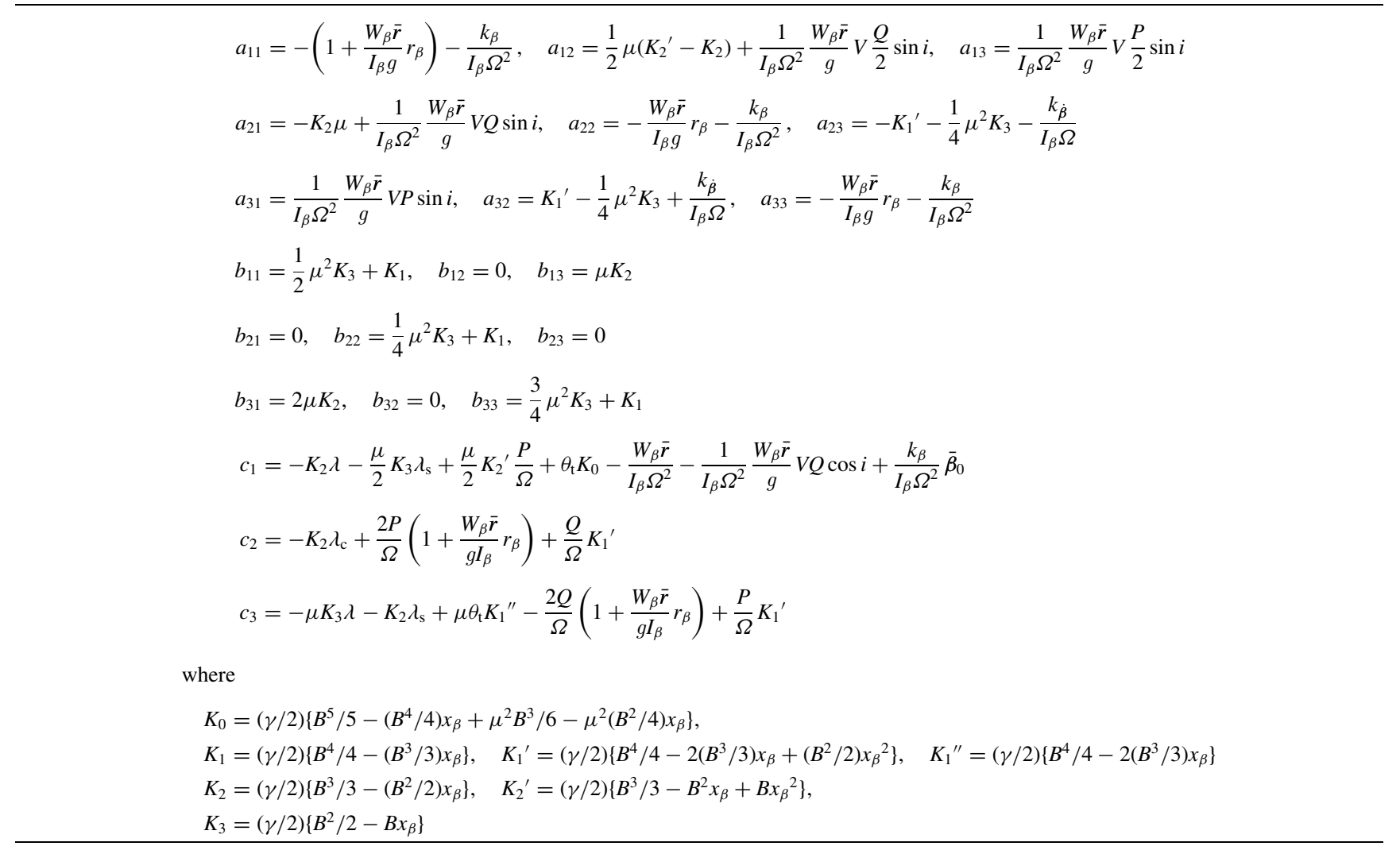

Table 3. Expressions of derivatives used for analyzing helicopter flight.

$$
\begin{aligned}
& \partial \beta_{\mathrm{c}} / \partial P=-\partial \beta_{\mathrm{s}} / \partial Q=\left\{\left(W_{\beta} \bar{r} r_{\beta} / g I_{\beta}+k_{\beta} / I_{\beta} \Omega^{2}\right)\left(1+W_{\beta} \bar{r} r_{\beta} / g I_{\beta}\right)(2 / \Omega)-\left(K_{1}{ }^{\prime}+k_{\dot{\beta}} / I_{\beta} \Omega\right)\left\{K_{1}{ }^{\prime}(1 / \Omega)\right\}\right\} \\
& \cdot\left\{\left(K_{1}{ }^{\prime}+k_{\dot{\beta}} / I_{\beta} \Omega\right)^{2}+\left(W_{\beta} \bar{r} r_{\beta} / g I_{\beta}+k_{\beta} / I_{\beta} \Omega^{2}\right)^{2}\right\}^{-1} \\
& \partial \beta_{\mathrm{s}} / \partial P=\partial \beta_{\mathrm{c}} / \partial Q=\left\{\left(K_{1}{ }^{\prime}+k_{\dot{\beta}} / I_{\beta} \Omega\right)\left(1+W_{\beta} \bar{r} r_{\beta} / g I_{\beta}\right)(2 / \Omega)+\left(W_{\beta} \overline{r_{\beta}} / g I_{\beta}+k_{\beta} / I_{\beta} \Omega^{2}\right)\left\{K_{1}{ }^{\prime}(1 / \Omega)\right\}\right\} \cdot\left\{\left(K_{1}{ }^{\prime}+k_{\dot{\beta}} / I_{\beta} \Omega\right)^{2}+\left(W_{\beta} \bar{r} r_{\beta} / g I_{\beta}+k_{\beta} / I_{\beta} \Omega^{2}\right)^{2}\right\}^{-1}>0 \\
& \partial \beta_{\mathrm{s}} / \partial \theta_{\mathrm{c}}=-\partial \beta_{\mathrm{c}} / \partial \theta_{\mathrm{s}}=\left\{K_{1}\left(K_{1}{ }^{\prime}+k_{\dot{\beta}} / I_{\beta} \Omega\right)\right\}\left\{\left(K_{1}{ }^{\prime}+k_{\dot{\beta}} / I_{\beta} \Omega\right)^{2}+\left(W_{\beta} \bar{r} r_{\beta} / g I_{\beta}+k_{\beta} / I_{\beta} \Omega^{2}\right)^{2}\right\}^{-1}>0 \\
& \partial \beta_{\mathrm{c}} / \partial \theta_{\mathrm{c}}=\partial \beta_{\mathrm{s}} / \partial \theta_{\mathrm{s}}=\left\{K_{1}\left(W_{\beta} \bar{r} r_{\beta} / g I_{\beta}+k_{\beta} / I_{\beta} \Omega^{2}\right)\right\}\left\{\left(K_{1}{ }^{\prime}+k_{\dot{\beta}} / I_{\beta} \Omega\right)^{2}+\left(W_{\beta} \bar{r} r_{\beta} / g I_{\beta}+k_{\beta} / I_{\beta} \Omega^{2}\right)^{2}\right\}^{-1}>0
\end{aligned}
$$

When $P=Q=0$, phase difference $D$ and gain $G$ between $\beta$ and $\theta$ are given by

$$
D=\tan ^{-1} \frac{K_{1}^{\prime}+k_{\dot{\beta}} / I_{\beta} \Omega}{W_{\beta} \bar{r} r_{\beta} / g I_{\beta}+k_{\beta} / I_{\beta} \Omega^{2}}
$$

and

$$
G=\frac{K_{1}}{\sqrt{\left(W_{\beta} \bar{r} r_{\beta} / g I_{\beta}+k_{\beta} / I_{\beta} \Omega^{2}\right)^{2}+\left(K_{1}{ }^{\prime}+k_{\dot{\beta}} / I_{\beta} \Omega\right)^{2}}} .
$$

Here, $\lambda_{\mathrm{c}}=\lambda_{\mathrm{s}}=0$ is assumed. When the assumptions $k_{\dot{\beta}}=$ 0 and $K_{1}=K_{1}{ }^{\prime}$ are also applied, the phase difference and amplitude ratio between $\beta$ and $\theta$ can be shown in one figure (Fig. 3 in Ref. 9). Note that the values of $D$ and $G$ are independent of the value of constant induced velocity $v_{0}$.

The derivatives for analyzing helicopter flight are obtained from Eqs. (13), (14) and (15), as shown in Table 3 . Note that the sign of $\partial \beta_{\mathrm{c}} / \partial P=-\partial \beta_{\mathrm{s}} / \partial Q$ depends on that of

$$
\begin{array}{r}
2\left(W_{\beta} \bar{r} r_{\beta} / g I_{\beta}+k_{\beta} / I_{\beta} \Omega^{2}\right)\left(1+W_{\beta} \bar{r} r_{\beta} / g I_{\beta}\right) \\
-\left(K_{1}{ }^{\prime}+k_{\dot{\beta}} / I_{\beta} \Omega\right) K_{1}{ }^{\prime} .
\end{array}
$$

\section{Results and Discussions}

The following were assumed in the calculations.

(1) The $k_{\dot{\beta}}$ was 0 .

(2) The lift coefficient of an airfoil was assumed by Eqs. (1a) and (1b) when Eq. (7) was solved. On the other hand, Eq. (1b) was assumed for any angle of attack when Eq. (11) was solved. This does not cause a large error, because the resultant angle of attack in a hover is larger than $10^{\circ}$ at almost all span-wise positions.

(3) The drag coefficient of an airfoil was set at 0.048 and 0 for solving Eq. (7) and Eq. (11), respectively.

(4) The induced velocity for the coaxial rotor, which is uniform on the actuator disk, $v_{0}$, was approximated as follows: The induced velocity for the isolated upper and lower rotor $\bar{v}_{0}$ was estimated by the simple momentum theory. Here, it is assumed that both the upper and lower rotors generate half of the total weight of the helicopter. The induced velocity $v_{0}$ was set to $\bar{v}_{0}$ and $2 \bar{v}_{0}$ for the upper and lower rotor, respectively. As stated above, the phase difference and gain between $\beta$ and $\theta$ calculated by Eq. (11) are independent of 
Table 4. Comparisons of gain $G=\Delta \beta / \Delta \theta$ of lower rotor between measurement and calculations.

\begin{tabular}{cccc}
\hline$\Delta \theta$ & Measurements & Calculation (Eq. (7)) & Calculation (Eq. (11)) \\
\hline $3.1^{\circ}$ & 0.85 & 0.84 & 0.78 \\
$7.1^{\circ}$ & 0.94 & 0.85 & 0.78 \\
\hline
\end{tabular}

the value of $v_{0}$. Then, the induced velocity for the upper and lower rotors was set roughly.

(A) Coning angle

For $k_{\beta}=7 \times 10^{-2} \mathrm{Nm}$, which was indicated above, the coning angle of the lower rotor was estimated by Eq. (7) and Eq. (11) as $\beta_{0} \approx 4.9^{\circ}$ and $\beta_{0} \approx 5.1^{\circ}$, respectively. These theoretical values of $\beta_{0}$ are close to the observed value.

(B) Flapping motion of lower rotors caused by cyclic pitch

The cyclic pitch at the blade root $\Delta \theta=\Delta \bar{\theta} \cos \left(\varphi_{1}-\right.$ $\left.315^{\circ}\right)\left(\Delta \bar{\theta}=3.1^{\circ}, 7.1^{\circ}\right)$ was input to the lower rotor. For these cyclic pitch, the flapping motions were observed as $\Delta \beta=\beta-\beta_{0}=\Delta \bar{\beta} \cos \left(\varphi_{1}-315^{\circ}-D\right)=\Delta \bar{\beta} \cos \left(\varphi_{1}-\right.$ $360^{\circ}$ ). The values of $D$ estimated by Eqs. (7) and (11) are also about $45^{\circ}$. The gain $G=\Delta \bar{\beta} / \Delta \bar{\theta}$ is shown in Table 4 . The theoretical values obtained by Eqs. (7) and (11) agree well. The differences between these theoretical values and the experimental ones are small.

(C) Flapping motion of upper rotors caused by pitching motion

(Case 1) In Ref. 8, the flapping motion of the stabilizer bar $\beta_{\mathrm{s}}$ is shown when the pitching motion of the fuselage $Q$ was $0.6 \mathrm{rad} / \mathrm{s}$. The motion was measured when the fuselage had made 5 revolutions around the pitching $(Y)$ axis, and it is terminal. The $\beta_{\mathrm{s}}$ is denoted by circles in Fig. 5 and it is approximated by $\beta_{\mathrm{s}}=19^{\circ} \cos \left(\varphi_{\mathrm{s}}+21^{\circ}\right)$.

The flapping motion of the upper rotor $\beta_{\mathrm{u}}$ during this pitching motion of the fuselage was newly measured. The motion is denoted by squares in Fig. 5 and it is approximated by $\beta_{\mathrm{u}}=4.4^{\circ}+9.2 \cos \left(\varphi_{\mathrm{u}}+42^{\circ}\right)$. The cyclic pitch to the upper rotor can be expressed ${ }^{11)}$ by $\Delta \theta_{\mathrm{u}}=15.2^{\circ} \cos \left(\varphi_{\mathrm{u}}+\right.$

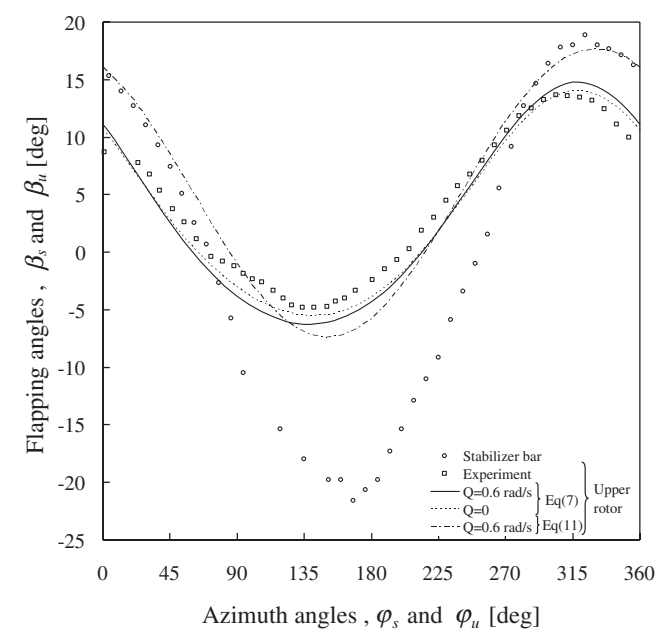

Fig. 5. Comparisons of terminal flapping motion of upper rotor between measurement and calculations. $Q=0.6 \mathrm{rad} / \mathrm{s}$. $62^{\circ}$ ) from the measured $\beta_{\mathrm{s}}$. The flapping motion of the upper rotor for this $\Delta \theta_{\mathrm{u}}$ was calculated by using Eq. (7) and Eq. (11). The flapping motions obtained by Eq. (7) and Eq. (11) are shown by the solid and dot-dash lines, respectively. The difference between these lines is negligibly small. The theoretical value obtained by Eq. (7) is approximated by $\beta_{\mathrm{u}}=4^{\circ}+11^{\circ} \cos \left(\varphi_{\mathrm{u}}+30^{\circ}\right)$. The phase difference between the theoretical $\beta_{\mathrm{u}}$ and the experimental value $\left(12^{\circ}\right)$ is observed and was the biggest discrepancy.

The broken line shows the flapping motion obtained by Eq. (7) when $Q=0$ and $\Delta \theta_{\mathrm{u}}=15.2^{\circ} \cos \left(\varphi_{\mathrm{u}}+62^{\circ}\right)$. The difference between the solid and broken lines is small and indicates that the flapping motion when $Q=0.6 \mathrm{rad} / \mathrm{s}$ and $\Delta \theta_{\mathrm{u}}=15.2^{\circ} \cos \left(\varphi_{\mathrm{u}}+62^{\circ}\right)$ is not caused by the pitching motion of fuselage $Q$ but by the input of cyclic pitch $\Delta \theta_{\mathrm{u}}$. Note that the input of cyclic pitch $\Delta \theta_{\mathrm{u}}$ is caused by the pitching motion of fuselage $Q$ via the flapping motion of the stabilizer bar.

(Case 2) The flapping motions of the stabilizer bar and the upper rotor when $Q=0.4 \mathrm{rad} / \mathrm{s}$ are shown in Ref. 11 . These motions were observed when the fuselage had made one revolution and they are not terminal. They are approximated by $\beta_{\mathrm{s}}=16^{\circ} \cos \left(\varphi_{\mathrm{s}}-20^{\circ}\right)$ and $\beta_{\mathrm{u}}=3.9^{\circ}+6.4^{\circ} \cos \left(\varphi_{\mathrm{u}}+\right.$ $\left.20^{\circ}\right)$. The cyclic pitch to the upper rotor is estimated by $\Delta \theta_{\mathrm{u}}=12.8^{\circ} \cos \left(\varphi_{\mathrm{u}}+21^{\circ}\right)$ from $\beta_{\mathrm{s}}$. The tip path plane of the upper rotor was inclined forward at this moment and it suppresses the pitching motion $Q$.

To investigate the effect of the value of $\zeta$ on flapping motion of the upper rotor, the flapping motions of the upper rotor in Case 1 and 2 were estimated when the $\zeta$ is not $41^{\circ}$ but $90^{\circ}$, with the following assumptions: (1) The flapping motion in Case 2 is determined mainly by the cyclic pitch as well as the terminal flapping motion in Case 1. (2) The flapping motion of the stabilizer bar is not affected by the upper rotor (the value of $\zeta$ ). The flapping motions of the upper rotor in Case 1 and 2 are shown in Table 5. When $\zeta=41^{\circ}$, the azimuth angle for the maximum $\beta_{\mathrm{u}}$ is closer to $0^{\circ}$ than when $\zeta=90^{\circ}$. Therefore, the pitching motion $Q$ can be more strongly suppressed by the upper rotor when $\zeta=41^{\circ}$.

In the above analyses $(\mathrm{A})-(\mathrm{C})$ for hovering flight, the theoretical results obtained by Eq. (7) agree well with those obtained by Eq. (11). The approximations included in Eq. (11) do not cause a large error for hovering flight. Moreover, the differences between these theoretical and experimental results are small. For hovering flight, the theoretical method based on Eqs. (7) and (11) can predict both the flapping motion of the upper rotor due to the cyclic pitch via the stabilizer bar, and that of the lower rotor due to input of cyclic pitch via the servo motors.

Table 5. Effect of $\zeta$ on $\beta_{\mathrm{u}}$.

\begin{tabular}{lcc}
\hline & $\zeta=41^{\circ}$ (Measured motions) & $\zeta=90^{\circ}$ (Estimated motions) \\
\hline Case 1 & $\beta_{\mathrm{u}}=4.4^{\circ}+9.2^{\circ} \cos \left(\varphi_{\mathrm{u}}+42^{\circ}\right)$ & $\beta_{\mathrm{u}}=4.4^{\circ}+9.2^{\circ} \cos \left(\varphi_{\mathrm{u}}+91^{\circ}\right)$ \\
Case 2 & $\beta_{\mathrm{u}}=3.9^{\circ}+6.4^{\circ} \cos \left(\varphi_{\mathrm{u}}+20^{\circ}\right)$ & $\beta_{\mathrm{u}}=3.9^{\circ}+6.4^{\circ} \cos \left(\varphi_{\mathrm{u}}+69^{\circ}\right)$ \\
\hline
\end{tabular}




\section{Conclusions}

The flapping motion of the rotors of a small coaxial helicopter was studied for hovering flight. This study clarified the meanings of the azimuth angle of cyclic pitch to the lower rotor and of the angle between the stabilizer bar and the upper rotor. The present helicopter is designed to increase ease of control and stability for hovering flight as follows: (i) The azimuth angle when cyclic pitch is input for the lower rotor is determined so that, for hovering flight, the lateral and longitudinal flapping motion can be controlled separately with two kinds of cyclic pitch inputs via the servo motors. (ii) The angle between the upper rotor and the stabilizer bar is determined so that, for hovering flight, the flapping motion caused by the cyclic pitch via the stabilizer bar decreases the attitude disturbance of the fuselage.

\section{References}

1) http://model2.hirobo.co.jp/

2) Sunada, S., Hirosue, W. and Kawashima, K.: Attitude Control of a Small Coaxial Helicopter with a Bell Type Stabilizer Bar, J. Jpn. Soc. Aeronaut. Space Sci., 52 (2004), pp. 494-498 (in Japanese).

3) Sunada, S., Tanaka, K. and Kawashima, K.: Maximization of ThrustTorque Ratio of a Coaxial Rotor, J. Aircraft, 42 (2005), pp. 570-572.

4) Gessow, A. and Myers, C. G., Jr.: Aerodynamics of the Helicopter, College Park Press, Bethesda, 1952, pp. 66-88.

5) Young, M. I.: A Simplified Theory of Hingeless Rotors with Applications to Tandem Helicopter, Proceeedings of the 18th Annual National Forum of AHS, 1962.

6) Harris, F. D.: Articulated Rotor Blade Flapping Motion at Low Advance Ratio, J. Am. Helicopter Soc., 17, 1 (1972), pp. 41-48.

7) Johnson, W.: Comparison of Calculated and Measured Helicopter Lateral Flapping Angles, J. Am. Helicopter Soc., 26, 2 (1981), pp. $46-50$

8) Ito, K., Nagashima, T. and Saotome, K.: An Experimental Study on the Cross Coupling Effects of a Helicopter Rotor in Low Advance Ratio Using the Dynamic Running Test Facility, J. Jpn. Soc. Aeronaut. Space Sci., 45 (1997), pp. 575-583 (in Japanese).

9) Azuma, A.: Dynamic Analysis of the Rigid Rotor System, J. Aircraft, 4, 3 (1967), pp. 203-209.

10) Azuma, A.: Aeronautic Engineering (I), Shokabo, Tokyo, 1989 (in Japanese).

11) Sunada, S., Kikuchi, A. and Tokutake, H.: A Study on a Bell Type Stabilizer Bar of a Small Coaxial Helicopter. Part II. Measurements of Its Flapping Motion, J. Jpn. Soc. Aeronaut. Space Sci, 54 (2006), pp. 272-275 (in Japanese). 